\title{
Anal atresia with transscrotal fistula
}

Accepted: 19 December 1994

\begin{abstract}
This report describes four infants born with an imperforate anus associated with a transscrotal fistula. This variant appears to be a low anomaly, but should be treated as an intermediate anorectal malformation.
\end{abstract}

Key words Anal atresia . Transscrotal fistula $\cdot$ Child

\section{Introduction}

The ultimate goal of operative correction of anal atresia is the creation of a normally functioning anus. For this purpose, it is important to make the distinction between low and high or intermediate anorectal anomalies. In cases of low anal atresia most pediatric surgeons recommend perineal anoplasty. Although recognition of the type of atresia may be difficult, in general anal atresia with a perineal fistula in boys is regarded as a low anomaly, suitable for local anoplasty.

Between January 1970 and December 1993, we treated 259 patients with anal atresia in the Pediatric Surgical Center Nijmegen, The Netherlands. In 4 boys there was a fistulous opening on the raphe of the penis, running through the scrotum and opening in the anorectum. After having erro-

C. Festen ( $)$ - R. S. V. M. Severijnen F. H. J. v.d. Staak - P. N. M. A. Rieu Pediatric Surgical Center, University Hospital Nijmegen, P. O. Box 9109, NL-6500 neously treated this as a low anomaly in the first patient, with a poor result, we considered the others as intermediate anomalies and performed a posterior sagittal anorectoplasty (PSARP) following a previous colostomy after birth. The functional results in these patients were simular to those in other cases of intermediate anorectal malformations.

\section{Case reports}

Case 1. A full-term male neonate (birth weight $3.8 \mathrm{~kg}$ ) was born with esophageal and anal atresias and a perineal fistula. The same day, the esophagus was repaired and the perineum explored in the belief that this was a low anomaly.

During operation, it was seen that the fistula passed through the scrotum and the rectum was found higher in the perineum. After cutting the fistula, the rectum had to be mobilized for anastomosis with the skin. The fistula itself was left in place and excised later. The child remained incontinent.

Case 2. A 2-months-old boy presented with anal stenosis after a perineal anoplasty was performed elsewhere for anal atresia. On inspection, a perineal fistula ran through the scrotum to the rectum associated with an anal stenosis (Fig. 1). A colostomy was performed; 2 months later re-exploration was done through a PSARP. The transscrotal fistula was cut and the rectal pouch mobilized and anastomosed with the skin after reconstruction of the pelvic floor. The fistulous tract was opened and the mucosa excised. There were good circular contractions around the anus after operation. Four months later the colostomy was closed. At the age of 5 years, there was mild stenosis of the anus and acceptable continence.

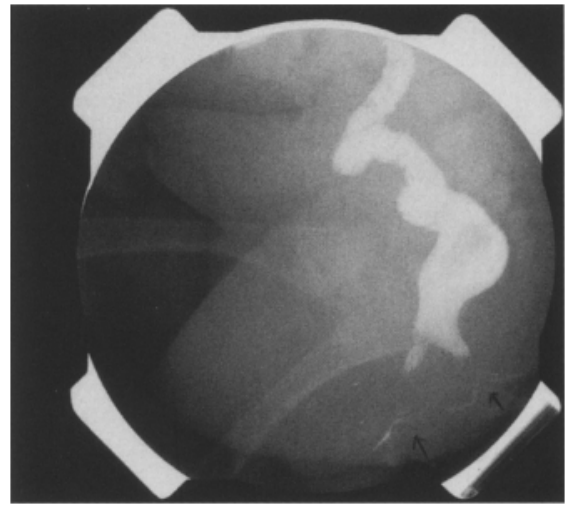

Fig. 1 Rectography showing transscrotal fistula anterior and anal stenosis posterior

Case 3. A male newborn (birth weight $3.3 \mathrm{~kg}$ ) presented with anal atresia. For a short distance on the raphe of the penis there was a fistulous tract stained by meconium (Fig. 2) and not in connection with the perineum, so that a transscrotal fistulous tract was presumed. A colostomy was performed. A loopogram confirmed an anal atresia of the intermediate type with a transscrotal fistula (Fig. 3). At the age of 6 months a PSARP was performed with partial excision of the fistula and mucosectomy. Two months later the colostomy was closed. At the age of 2 years, he had good anal function with spontaneous bowel movements.

Case 4. A male neonate (birth weight $3 \mathrm{~kg}$ ) was born with anal atresia and a perineal fistula. On exploration the next day, a transscrotal fistula was identified and no further exploration was performed. A colostomy was done and 6 months later he underwent a PSARP with transection of the transscrotal fistula and excochleation of the mucosa. Two months later the colostomy was closed. At the age of $11 / 2$ years, he had good anal function with spontaneous bowel movements. 


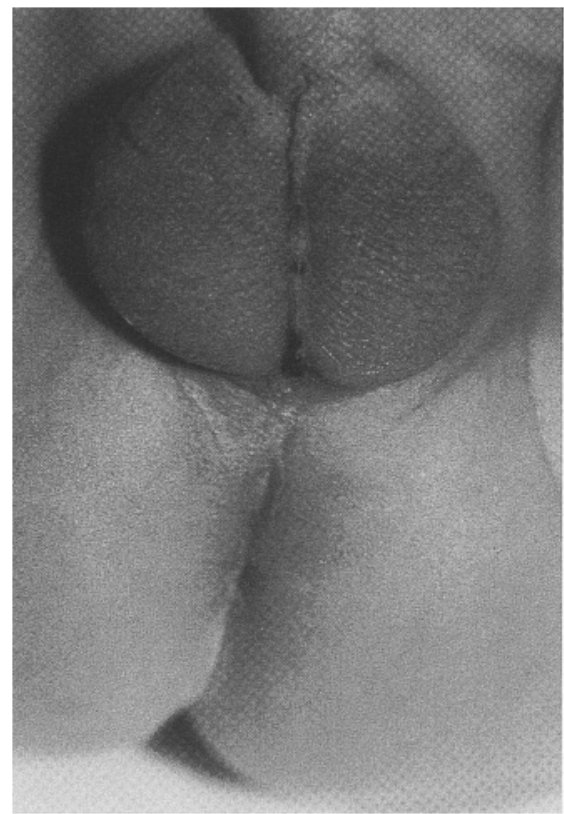

Fig. 2 Perineum showing subcutaneous scrotal fistula stained with meconium and anal atresia

\section{Discussion}

In general, a male patient with anal atresia and a perineal fistula is regarded as having a low anorectal malformation and most often treated by perineal anoplasty. In our series we found four patients who appeared to have low anal atresia with a perineal fistula that proved to be at least an intermediate type with a transscrotal fistula. The fistula runs through the scrotum parallel to the urethra from the rectal pouch to the raphe of the penis; from there it may follow the raphe subcutaneously for some distance.

In the pediatric surgical literature we found only two reports of anal atresia with a transscrotal fistula, one as a personal communication of Nixon in the monograph of Stephens and Smith [4] and one in a report of a Japanese study group of anorectal anomalies [3]. In the Japanese report this was called a recto-penile fistula, and there was a picture of a radiograph of the fistulous tract running parallel to the urethra. Currarino [2] described two patients with imperforate anus associated with a recto-bulbar-cutaneous fistula and quoted a simular case of Asano et al. [1] where the rectocutaneous fistula was associated with a communication in the midportion with the bulbar urethra.

Recently, Shanbhogue et al. [5] reported three male newborns with recto-perineal fistulae, but did not give an exact description of the anatomy. They suggested these were genuine perineal fistulae with a high rectal pouch.

Our four cases demonstrate that anal anomalies in boys with a perineal fistula deserve more attention and evaluation. When there is a true transscrotal fistula, the anomaly is better treated as a high or intermediate anal atresia in order not to destroy essential structures by intensive exploration. This policy may guarantee better functional results.

In case of doubt, it is wiser to do a colostomy and perform contrast studies later on to identify the actual anatomy. This is especially important since the functional results of PSARP in intermediate anomalies are as a rule quite acceptable and these children can be saved a life of misery. The fistulous tract can be excised, but we unroofed the fistula as far as seemed warranted and did a mucosectomy in the remainder of the tract. These residual fistulous tracts obliterated spontaneously.

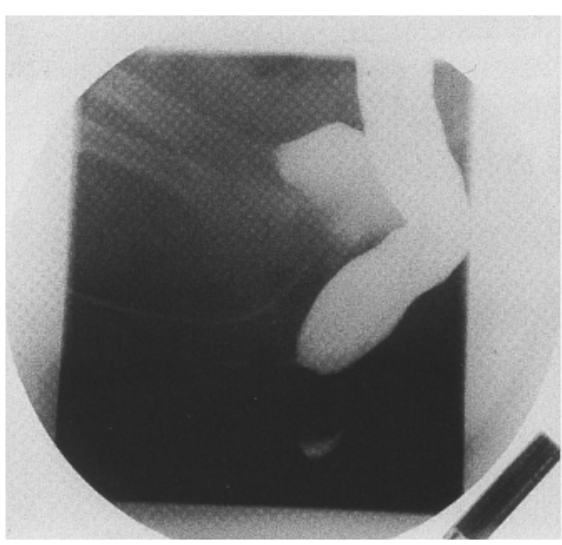

Fig. 3 Loopogram and urethrocystography showing urethra and transscrotal fistula

The intermediate type of anal atresia with a transscrotal fistula should be incorporated into the international classification of the Melbourne group.

\section{References}

1. Asano S, Kitatani H, Konuma K (1983) A newborn with a covered anus complicated by two concomitant unique fistulas. Z Kinderchir 38: 258-261

2. Currarino G (1994) Imperforate anus associated with a recto-bulbar-cutaneous fistula. J Pediatr Surg 29: 102-105

3. Japan Study Group of Anorectal Malformations (1982) A group study for the classification of anorectal anomalies in Japan with comments to the international classification (1970). J Pediatr Surg 17: $302-308$

4. Nixon HH (1970) Personal communication. In: Stephens FD, Smith ED (eds) Anorectal malformations in children. Update 1988. Alan R Liss, New York, p 60

5. Shanbhogue LKR, Langemeijer RATM, Madern GC, Molenaar JC (1994) Rectoperineal fistula in newborn boys. $J$ Pediatr Surg 29: 536-537 